\title{
Study on the Immunogenicity of Three Recombinant Lactic Acid Bacteria Expressing ASFV p14.5 Protein
}

Quntao Huang ( $\nabla 10987188 @ q q . c o m$ )

Jilin Agricultural University

Tian-Ming Niu

Jilin Agricultural University

Bo-Shi Zou

Jilin Agricultural University

Jun-Hong Wang

Jilin Agricultural University

Jun-Hong Xin

Jilin Agricultural University

Hui Niu

Jilin Agricultural University

Nan Li

Jilin Agricultural University

Yu-Xin Jiang

Jilin Agricultural University

Jun-Fu Bao

Jilin Agricultural University

Di Zhang

Jilin Agricultural University

Xi-Ze Feng

Jilin Agricultural University

Ting-Ting Sun

Jilin Agricultural University

\section{Xin Wang}

Jilin Agricultural University

Kai-Dian Yang

Jilin Agricultural University

Ying Wang

Jilin Agricultural University

Dan-Dan Zhao

Jilin Agricultural University

Gui-Lian Yang 
Jilin Agricultural University

Chun-Feng Wang

Jilin Agricultural University

Original article

Keywords: ASFV, p14.5, CTA1-DD, IL-33, L. plantarum, Immune evaluation

Posted Date: November 12th, 2021

DOI: https://doi.org/10.21203/rs.3.rs-1012939/v1

License: (c) (1) This work is licensed under a Creative Commons Attribution 4.0 International License. Read Full License 


\section{Abstract}

The African Classical Swine Fever Virus (ASFV) has spread severely all over the world. The lack of vaccines has dealt a heavy blow to the pig industry.In this study, the p14.5 protein encoded by the African swine fever virus was used as the antigen, and the p14.5 protein gene was expressed in vitro using the Lactobacillus expression system. Three new functional recombinant Lactobacillus plantarum((L. plantarum) were constructed and the p14.5 was successfully detected using western technology.Protein,

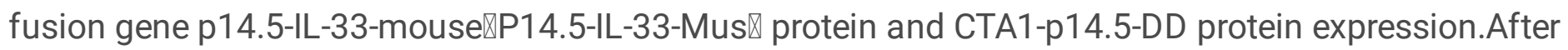
oral immunization of SPF mice with recombinant lactic acid bacteria, flow cytometry and ELISA were used to detect that the differentiation and maturity of $T, B$, and DC cells of the mice were higher than those of the control group, and specific antibodies were produced. In contrast, the immune effect of the adjuvant group was stronger than that of the single antigen group, and the IL-33 adjuvant effect was stronger than that of the CTA1-DD adjuvant. This study provides effective data support for the prevention of African swine fever virus infection with new lactic acid bacteria preparations, and has certain innovative significance.

\section{Introduction}

African Swine Fever (ASF) is an acute, thermal, and highly exposed infectious disease in pigs caused by the African swine fever virus (ASFV). Clinical symptoms and pathological changes are similar to classic swine fever, which is easy to be misdiagnosed in clinical diagnosis, specifically manifested as high fever, skin congestion, abortion, edema and organ bleeding and the mortality rate is as high as $100 \%$ (Bonnet, et al. 2020). The disease infects only pigs and has been endemic in over 30 countries and the disease is listed as a must-notified animal disease by OIE(Sauter-Louis, et al. 2021).

At present, African swine fever vaccine is prepared in three main ways: first, inactivated vaccine;second,subunit vaccine and live vector vaccine; third, recombinant virus vaccine(Rock 2017). The first of these is the most common and direct method for preparing viral vaccines,but African swine virus encodes proteins such as immunosuppression, immune tolerance, and antibody-dependent enhancing effects (ADE)(Wang, et al. 2020). Therefore, inactivated vaccine immunity cannot provide effective immune protection for pigs. Therefore, subunit vaccines and genetic recombinant vaccines may be better subjects(Wang, et al. 2021).The entire genome of ASFV contains 167open reading frames (ORFs), which can encode 150 - 200 proteins, approximately 50 of which are structural proteins(Xian and Xiao 2020), and the p14.5 protein is one of the structural proteins of the ASFV.The structural protein p14.5, a key virulence factor and late expression protein of ASFV, can block its recruitment by binding to the IRF3 and plays an important role in inhibiting IRF3 phosphorylation and interferon production. This topic the pE120R gene encoding the p14.5 protein was selected as the research focus(Liu, et al. 2021).

CTA1-DD is an artificial adjuvant composed of CTA1 subunit of cholera toxin enzyme activity and D domain dimer of Staphylococcus aureus protein A(Eliasson, et al. 2011).This molecule has been previously demonstrated in mice and non-human primates to be non-toxic and is a safe adjuvant.It is 
able to stimulate robust and balanced CD4+T cell responses, greatly enhancing specific antibody production.After systemic and mucosal immunity, follicular dendritic cells $₫$ FDCs $₫$ in peripheral lymph nodes to increase germinal center B cells and Tfh responses(Schussek, et al. 2020).IL-33, a multifunctional gene discovered in 2005 , is secreted in case of cell damage and plays a role in regulating immunity(Ochayon, et al. 2020). It can respond directly with DC to stimulate the differentiation of naive T cells into Th2 or helper T cells(Matta, et al. 2014). It was demonstrated that during viral infection, IL-33 can help with virus clearance by promoting the expansion of NK and NKT cells and enhancing Th1 and CD8+T cell responses(Kim, et al. 2019).

Lactobacillus is an essential and important physiological flora in the human body. It is widely found in the human gut, regulating the human intestinal health, and is directly related to people's health and longevity.Lactobacillus bacteria as a carrier presenting biomolecules to the gastrointestinal tract for the treatment and prevention of diseases is increasingly mature, and itself has obvious advantages, and can stimulate the body to produce an effective and long-term immune response.S-layer protein is a singlemolecule crystal structure protein on the outermost surface of a cell. It is considered to be the outermost structure of the cell membrane in many archaea(Peirotén and Landete 2020). The surface of this protein is used to display foreign proteins. The S-layer protein derived from Lactobacillus not only has the ability to anchor and express foreign proteins, but also has adhesive properties, and can also be used as an adjuvant to stimulate the body's immune response(Klotz, et al. 2020).In this study $\mathbb{W} W$ use the vector pLPS (pLp_1261 Inv derivative, where Ip_1261 and Inv fusion gene has been replaced by the SP-linkerS_anchoring sequence of SIpA gene containing MCS1), which can anchor the expression protein in the S layer, and insert p14.5 and p14. The 5-IL-33-Mus and CTA1-p14.5-DD gene sequences were constructed, and three new plasmids were constructed and transformed into Lactobacillus plantarum NC8 to construct three recombinant lactic acid bacteria.Through animal experiments, evaluate the immune effects of three strains of lactic acid bacteria, laying a theoretical foundation for subsequent research on vaccines and vaccine adjuvants.

\section{Material And Methods}

\subsection{Animals and ethical statement}

The animals used in this experiment were purchased from HFKBioscience Co., Ltd. (Beijing, China). Pathogen-free female C57BL/ 6 mice aged 6 to 8 weeks were raised in SPF rooms. The whole animalexperiment met the requirements of the Animal Management and Ethics Committee of Jilin Agricultural University.

\subsection{Construction of recombinant $L$. plantarum}

The base sequence of ASFV E120R gene (gene number: 41901181) and IL-33-Mus (gene number: AY905582.1) gene base sequence were obtained through NCBl,and the base sequence of CTA1 and DD gene was obtained byconsulting the literature,which was optimized and synthesised by Nanjing Genscript synthesis.The p14.5 gene,p14.5-IL-33-Mus gene and CTA1-p14.5-D-D gene were cloned into pLP-S 
vector. The constructed plasmidwas transformed into Lactobacillus plantarum NC8(CCUG61730).Three recombinant Lactobacillus plantarumNC8-pLP-S-p14.5, NC8-pLP-S-p14.5-IL-33-Mus and NC8-pLP-SCTA1-p14.5-D-D were obtained. They were sequenced and identified by Comate Bioscience Co., Ltd. (Changchun, China).

\subsection{Preparation of $\mathrm{p} 14.5$ protein}

Insert p14.5 gene sequence into pET28a expression vector, construct plasmid pET-28a-p14.5, transform the plasmid into E. coli competent BL21, and obtain recombinant E. coli BL21-pET-28a-p14.5. IPTG (100 $\mathrm{MM}$ ) induces protein expression, the cells are sonicated and the protein is collected in the inclusion body, and the 14.5 protein is recovered after purification.

\subsection{Western blotting}

Put the preserved bacteria liquid NC8-pLP-S-p14.5, NC8-pLP-S-p14.5-IL-33-Mus and NC8-pLP-S-CTA1p14.5-DD from $-80^{\circ} \mathrm{C}$ in the refrigerator Take it out, inoculate it in $5 \mathrm{~mL}$ of MRS liquid containing Erm $(5 \mu \mathrm{g} / \mathrm{mL})$, and incubate overnight. The next day, transfer to $200 \mathrm{~mL}$ and add Erm $(5 \mu \mathrm{g} / \mathrm{mL})$ to the MRS liquid. After culturing at $37^{\circ} \mathrm{C}$ anaerobic workstation with od500 $=0.3$, add $125 \mu \mathrm{L}(50 \mathrm{ng} / \mathrm{mL})$ of sakacin $\mathrm{P}$ inducer (SppIP), and induce culture for $8 \mathrm{~h}$. After centrifugation, the precipitate was ultrasonically broken, and the Western experiment was performed after processing the sample. The results were analyzed using Amersham Imager (General Electric Company) to test protein expression.

\subsection{Immunization}

Fifty mice were randomly divided into 5 groups with 10 mice in each group. Feed PBS, NC8-pLP-S, NC8pLP-S-p14.5, NC8-pLP-S-p14.5-IL-33-Mus, NC8-pLP-S-CTA1-p14.5-DD, respectively , Each feeding $200 \mu \mathrm{L}$ $(1 \times 109 \mathrm{CFU} / \mu \mathrm{L})$, mark the right ear of each mouse, and each group is marked with 10 natural digital numbers. The new functional recombinant lactic acid bacteria were fed to mice by oral administration. The first immunization time was the first, second, and third day, with an interval of one week, and the second immunization was performed on the 11th, 12th, and 13th day, with an interval of one week. Three immunizations were performed on the 21st, 22nd, and 23rd day. After the first immunization and the third immunization, three mice from each group were randomly selected for flow cytometry experiments. Mouse feces and serum were collected on days 0, 9, 19, and 29 for enzyme-linked immunosorbent assay (ELISA) detection.

\subsection{Single cell suspension preparation method}

In the ultra-clean table, use ophthalmic scissors and ophthalmic forceps (autoclaved) to peel off the mesenteric lymph nodes (MLNs), Pey's collective lymph nodes (PPs) and the spleen, remove excess fat, and place on ice. Place the folded 200 mesh sterile filter in a sterile small plate, and add $1 \mathrm{~mL}$ of RPMI1640 complete culture solution. Put the spleen into the strainer and gently grind it with the end of a sterile $1 \mathrm{~mL}$ syringe until the grind is complete, then suck the liquid into a $1.5 \mathrm{~mL}$ EP tube and place it in a precooled centrifuge at $4^{\circ} \mathrm{C}, 2000 \mathrm{rpm}$, and centrifuge for $5 \mathrm{~min}$. Then discard the supernatant, add $0.5 \mathrm{~mL}$ of 
red blood cell lysate, lyse on ice for 3 minutes, add $0.5 \mathrm{~mL}$ of PBS and mix for 2 minutes. Centrifuge at $2000 \mathrm{rpm}, 4^{\circ} \mathrm{C}$ for $5 \mathrm{~min}$, and discard the supernatant. Wash with $1 \mathrm{~mL}$ PBS once, discard the supernatant and add $1 \mathrm{~mL}$ of complete medium. Count on a cell counting plate after diluting 100 times. MLN and PP knots are treated in the same way, but there is no need to lyse with red blood cell lysate. After treatment, it was diluted 20 times and counted.

\subsection{Flow cytometry}

Use antibody staining, use antibody B220, IgA, CD11C, CD80\CD86 to stain PPs and antibody CD3, 4, 8, IL-4, IFN- $y$ to stain Single-cell suspensions of spleen (SP) according to the instructions and MLNs.All antibodies are sourced from BD Biosciences, USA.BD fluorescence-activated cells were sorted and analyzed by FACS in an LSRFortessa analyzer (BD Bioscience, USA). All data were analyzed using FlowJo 7.6 software.

\subsection{Enzyme-linked immunosorbent assay}

Detect secreted SIgA in stool and IgG in serum.

Coating: Coat each sample well of a 96-well culture plate with purified P14.5 antigen (concentration of $1 \mu \mathrm{g} / \mathrm{mL}), 100 \mu \mathrm{L}$ per well, and let it stand overnight at $4^{\circ} \mathrm{C}$. Washing: Wash each sample well with PBS containing $0.05 \%$ Tween- 20 at room temperature, $300 \mu \mathrm{L} /$ well, once every $5 \mathrm{~min}$, a total of three times.Blocking: add PBS containing $1 \% \mathrm{BSA}$ to each sample well, block overnight at $4^{\circ} \mathrm{C}$, $150 \mu \mathrm{L} /$ well.Washing: Repeat step 2.Primary antibody: Add the sample to be tested (sample treated with feces and serum), and seal the membrane.Washing: Repeat step 2.Secondary antibodies: The secondary antibodies are diluted anti-mouse SIgA and anti-mouse IgG, sealed with membrane.Washing: Repeat step 2.Develop the color of the substrate, add $100 \mu \mathrm{L} /$ well of the color developing solution, and react for $1 \mathrm{~h}$ in the dark at $37^{\circ} \mathrm{C}$.Stop reaction: add stop solution $10 \% \mathrm{H} 2 \mathrm{SO} 4,50 \mu \mathrm{L} /$ well. The detection result of the microplate reader, the detection wavelength is $450 \mathrm{~nm}$, the plate is $30 \mathrm{~s}$, and the data is retained.

\subsection{Analyze the results.Statistical analysis}

All the data in the experiment came from at least three independent experiments and are expressed as the average \pm SEM. GraphPad Prism 5.0 software was used to test the differences. $P<0.05$ was considered to represent a significant difference. Analysis of variance (ANOVA) with T ukey's multiple comparison test was used to evaluate the significance.

\section{Results}

3.1 Construction of plasmids and expression of target genes in vitro

Three new functional recombinant lactic acid bacteria were successfully constructed(Fig. 1A,Fig. 1B,Fig. 1C) . and the expression of p14.5 protein, fusion gene p14.5-IL-33 protein and CTA1-p14.5-D-D protein were successfully detected using western technology(using his tag as the detection antigen )(Fig. 1D) 
.The WB result shows that the target band is consistent with the expected size, which proves Recombinant Lactobacillus plantarum successfully expressed foreign protein.

\subsection{Recombinant Lactobacillus plantarum enhances the cellular immunity of mice}

The spleen is the main peripheral immune organ, the place where T cells and B cells settle, and it is also the main organ that produces antibodies in the body. It plays an important role in the body's defense and immune response. Lymph nodes are highly specialized lymphatic organs. After foreign bodies such as bacteria invade the body, the local lymph nodes enlarge, produce an immune response, and proliferate a large number of lymphocytes. CD3, CD4, and CD8 are the surface markers of T lymphocytes, which can mark cytotoxic $\mathrm{T}$ cells and helper T cells. IFN- $\gamma$ has anti-viral, anti-tumor and immune regulation effects. It can also promote NK cell activity, promote antigen presentation and increase macrophage lysosomal activity. Recombinant Lactobacillus plantarum enhanced the immune effect of the body. The content of CD3+CD8+T cells in CD3+CD4+T cells in lymphocytes of the spleen and mesenteric lymph nodes increased relatively(Fig. 2A囚Fig. 2B), and at the same time, the content of IFN-y also increased significantly.(Fig. 2C区Fig. 2D).

\subsection{Recombinant Lactobacillus plantarum enhances mucosal immunity in mice}

$\mathrm{PP}$ is a secondary lymphoid organ of the host's small intestine, which gathers various cells such as B cells and $\mathrm{M}$ cells. Among them, $\mathrm{M}$ cells play an important role in the uptake of antigens. PP exists from the mucosa to the submucosa, B cells are located in the germinal center, and $\mathrm{T}$ cells are around it. Among them, PP undertakes the task of monitoring the mucosal immune response of intestinal cavity antigens. B220 and IgA are B cell activation markers, so the level of activated B cells in PP is an important indicator for evaluating the body's immunity.Recombinant Lactobacillus plantarum significantly improved the detection of B220+lgA+ cells in PPs (Fig 2E). The results of SlgA detection in mouse feces showed that with the increase in the number of immunizations, NC8-pLP-S-p14.5, NC8-pLP-S-p14.5-IL-33-Mus and NC8-pLP-S-CTA1-p14 The content of SIgA in the feces of .5-DD mice gradually and significantly increased. After the third immunization, fed mice with NC8-pLP-S-p14.5, NC8-pLP-S-p14.5-IL-33-Mus and NC8-pLP-S-CTA1-p14.5-DD The content of IgA in feces was significantly higher than that of mice fed PBS, and there was no significant change in the content of SIgA in feces of mice in the PBS group and the empty carrier NC8-pLP-S group (Fig 3).

\section{Discussion}

African swine fever has caused huge economic losses to the pig breeding industry around the world. Although the research on non-pest vaccines began in the 1960s, as of now, there is no effective non-pest vaccine on the market in the world. As there is no vaccine or therapeutic drug that can effectively prevent African swine fever in the world, all sick pigs are currently culled(Arias, et al. 2017).Therefore, the research on the ASF subunit vaccine is of great significance(Turlewicz-Podbielska, et al. 2021). 
The use of lactic acid bacteria as a delivery vector to construct genetically engineered vaccines is already a common method to fight viruses(Mojgani, et al. 2020). Usually, the gene sequence of the core protein of the virus is selected, the appropriate epitope gene is combined with the vector that can express the epitope, and the appropriate adjuvant is used to construct the plasmid to transform into the lactic acid bacteria as a virus vaccine. As a kind of probiotics, lactic acid bacteria have irreplaceable safety advantages compared with other bacterial delivery systems such as Salmonella(Takahashi, et al. 2019). They can activate the body's cellular immunity, humoral immunity, and mucosal immunity at the same time, and have great potential(Lycke 2012).

Lactobacillus plantarum has a very good prospect as a mucosal vaccine vector. Yang constructed a recombinant Lactobacillus NC8 expressing influenza fusion genes HA2 and 3M2e. After immunization with mice and H9N2 and $\mathrm{H} 1 \mathrm{~N} 1$ challenge tests, the results showed that Lactobacillus plantarum can provide effective Protect, while reducing the extent of lung disease(Bo, et al. 2019). In 2018 Huang and 2017 Yanlong Jiang et al. successfully expressed porcine epidemic diarrhea virus S gene and avian influenza virus H9N2 subtype HA2 protein with pSIP409-pgsA' anchored expression vector, and obtained oral immunization of engineered bacteria Chicks have a moderate anti-coccidial effect(Yang, et al. 2017). Anchoring the Lactobacillus plantarum containing HA2 protein and the adjuvant molecule LTB can significantly improve the anti-avian influenza virus ability of immunized mice.

Studies have proved that exogenous IL-33 can enhance the antiviral protection of influenza virus infection. Exogenous IL-33 induces the recruitment of dendritic cells, increases the secretion of the proinflammatory cytokine IL-12, and promotes cytotoxic T cell responses in the local microenvironment(Yang, et al. 2011). At the same time, there is a large amount of evidence that IL-33 has an anti-virus effect, and, because IL-33-Mus and IL-33-Pig have very high homology, this experiment uses IL-33 as a potential mucosal adjuvant. The research provides a preliminary reference for future experiments with pigs.

We successfully constructed three new functional recombinant plants NC8-pLP-S-p14.5, NC8-pLP-Sp14.5-IL-33-Mus and NC8-pLP-S-CTA1-p14.5-DD Lactobacillus, and verified their successful expression in vitro. Because lactic acid bacteria can colonize in the mouse intestine, and can stimulate lactic acid bacteria to express $\mathrm{p} 14.5$ protein and p14.5-IL-33, CTA1-p14.5-DD fusion protein in the intestinal environment, the body's T cells recognize p14 .5 protein so that specific antibodies can be produced. Preliminary animal experiments with mice showed that feeding recombinant lactic acid bacteria can improve the immune effect of mice to a certain extent.

From the flow cytometry experiment, it can be seen that the secretion of B220 and IgA and the contents of $11 C+C D 80$ and $11 C+C D 86$ in the PP nodal lymphocytes of the mice fed the recombinant lactic acid bacteria group are greater than those of the control group, and the adjuvant group can promote more $B$ cells secrete specific antibodies and at the same time promote the differentiation of more DC cells; the spleen and mesenteric lymph nodes of mice fed with recombinant lactic acid bacteria have more $\mathrm{CD} 3+\mathrm{CD} 4+$ and $\mathrm{CD} 3+\mathrm{CD} 8+$ than the control group, and the adjuvant group is larger than the single The 
antigen group proved that the recombinant lactic acid bacteria promoted the differentiation of mouse $T$ cells, and the adjuvant group had a better promotion effect. The expression of CD4+IFN- $y$ and CD8+IFN$Y$ in the spleen and mesenteric lymph nodes of mice fed with recombinant lactic acid bacteria group increased significantly, and the increase in adjuvant group was higher than that in single antigen group. The Elisa experiment was used to detect the specific SIgA content in mouse feces, which proved that the recombinant lactic acid bacteria group stimulated the mouse body to produce specific antibodies, and the secretion of the adjuvant group was higher than that of the single antigen group, which proved the adjuvant's immune support ability.

The above series of experimental results all show that when fed with new functional recombinant lactic acid bacteria, the immunity of mice is significantly improved. Recombinant Lactobacillus plantarum can improve the humoral immunity, cellular immunity and mucosal immunity of mice, laying a theoretical foundation for the development of oral ASFV vaccine. For the phenomenon of ASFV infection through mucosa, mucosal vaccine may have a certain effect of blocking the virus. This experiment provides a certain theoretical guidance for subsequent vaccine research.

\section{Abbreviations}

African Classical Swine Fever Virus (ASFV)

Lactobacillus plantarum((L. plantarum)

African Swine Fever (ASF)

open reading frames (ORFs)

follicular dendritic cells $\mathbb{F D C s \rrbracket}$

mesenteric lymph nodes (MLNs)

Pey's collective lymph nodes (PPs)

spleen (SP)

p14.5-IL-33-mouse『P14.5-IL-33-Mus $\bigotimes$

\section{Declarations}

\section{Ethics approval and consent to participate}

The whole animal experiment met the requirements of the Animal Management and Ethics Committee of Jilin Agricultural University.

\section{Consent for publication}


Not applicable.

\section{Availability of data and materials}

The raw data reported in the present paper have been deposited in Jilin Agricultural University, Chang Chun, China.

\section{Competing interests}

The authors declare that there are no competing interests.

\section{Funding}

This work was supported by the National Key Research and Development Program of China (2017YFD0501000, 2017YFD0501200, 2017YFD0500400), the National Natural Science Foundation of China $(32072888,31972696,31941018,32072897)$ and Science and Technology Development Program of Jilin Province (20180201040NY, 20190301042NY).

\section{Authors' contributions}

Dan-Dan Zhao, Qun-Tao Huang and Chun-Feng Wang conceived and designed research. Tian-Ming Niu and Qun-Tao Huang conducted experiments. Bo-Shi Zou and Gui-Lian Yang analyzed data. Qun-Tao Huang wrote the manuscript.Jun-Hong Wang,Jun-Hong Xin,.Hui Niu ,Nan Li ,Yu-Xin Jiang,Jun-Fu Bao,Di Zhang,.Xi-Ze Feng,Ting-Ting Sun ,Xin Wang ,Kai-Dian Yang,Ying Wang Contributed to the work. All authors read and approved the manuscript.

\section{Acknowledgements}

We thank Chun-Feng Wang and Dan-Dan Zhao for their support in the experiments.

\section{References}

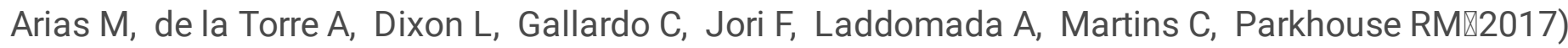
Approaches and Perspectives for Development of African Swine Fever Virus Vaccines. Vaccines 5(4).

Bo F, Yang WT, Shonyela SM, Jin YB, Huang KY, Shao LN, Wang C, Zhou Y, Li QY, Jiang YL (2019区 Immune responses of mice inoculated with recombinant Lactobacillus plantarum NC8 expressing the fusion gene HA2 and 3M2e of the influenza virus and protection against different subtypes of influenza virus. Virus research 263:64-72.

Bonnet SI, Bouhsira E, De Regge N, Fite J, Etoré F, Garigliany MM, Jori F, Lempereur L 『2020) Putative Role of Arthropod Vectors in African Swine Fever Virus Transmission in Relation to Their Bio-Ecological Properties. Viruses 12(7). 
Eliasson DG, Helgeby A, Schön K, Nygren C, El-Bakkouri K, Fiers W, Saelens X, Lövgren KB $₫ 2011)$ A novel non-toxic combined CTA1-DD and ISCOMS adjuvant vector for effective mucosal immunization against influenza virus. Vaccine 29(23):3951-61.

Kim CW, Yoo HJ, Park JH, Oh JE, Lee HK囚2019) Exogenous Interleukin-33 Contributes to Protective Immunity via Cytotoxic T-Cell Priming against Mucosal Influenza Viral Infection. Viruses 11(9).

Klotz C, Goh YJ, O'Flaherty S, Barrangou R囚2020『S-layer associated proteins contribute to the adhesive and immunomodulatory properties of Lactobacillus acidophilus NCFM. BMC microbiology 20(1):248.

Liu H, Zhu Z, Feng T, Ma Z, Xue Q, Wu P, Li P, Li S, Yang F, Cao W, Xue Z, Chen Hヌ2021区African swine fever virus E120R protein inhibits interferon- $\beta$ production by interacting with IRF3 to block its activation. Journal of virology:JVI0082421.

Lycke, N囚2012『Recent progress in mucosal vaccine development: potential and limitations. Nature reviews. Immunology 12(8):592-605.

Matta BM, Lott JM, Mathews LR, Liu Q, Rosborough BR, Blazar BR, Turnquist HR 2014) IL-33 is an unconventional Alarmin that stimulates IL-2 secretion by dendritic cells to selectively expand IL33R/ST2+ regulatory T cells. Journal of immunology (Baltimore, Md. : 1950) 193(8):4010-20.

Mojgani, N, Y Shahali, and M Dadar $₫ 2020$ Immune modulatory capacity of probiotic lactic acid bacteria and applications in vaccine development. Beneficial microbes 11(3):213-226.

Ochayon DE, Ali A, Alarcon PC, Krishnamurthy D, Kottyan LC, Borchers MT, Waggoner SN『2020区IL-33 promotes type 1 cytokine expression via p38 MAPK in human NK cells. Journal of leukocyte biology 107(4):663-671.

Peirotén, Á, and JM Landete $₫ 2020 \bigotimes N$ atural and engineered promoters for gene expression in Lactobacillus species. Applied microbiology and biotechnology 104(9):3797-3805.

Rock, DL囚2017囚Challenges for African swine fever vaccine development-"... perhaps the end of the beginning.". Veterinary microbiology 206:52-58.

Sauter-Louis C, Conraths FJ, Probst C, Blohm U, Schulz K, Sehl J, Fischer M, Forth JH, Zani L『2021》 African Swine Fever in Wild Boar in Europe-A Review. Viruses 13(9).

Schussek S, Bernasconi V, Mattsson J, Wenzel UA, Strömberg A, Gribonika I, Schön K, Lycke NYヌ2020) The CTA1-DD adjuvant strongly potentiates follicular dendritic cell function and germinal center formation, which results in improved neonatal immunization. Mucosal immunology 13(3):545-557.

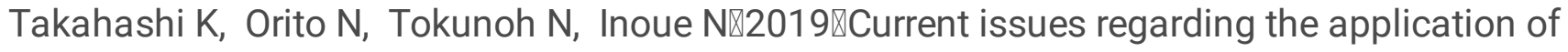
recombinant lactic acid bacteria to mucosal vaccine carriers. Applied microbiology and biotechnology 103(15):5947-5955. 
Turlewicz-Podbielska H, Kuriga A, Niemyjski R, Tarasiuk G, Pomorska-Mól Mヌ2021®African Swine Fever Virus as a Difficult Opponent in the Fight for a Vaccine-Current Data. Viruses 13(7).

Wang F, Zhang H, Hou L, Yang C, Wen Y区2021هAdvance of African swine fever virus in recent years. Research in veterinary science 136:535-539.

Wang T, Sun Y, Huang S, Qiu HJ囚2020冈Multifaceted Immune Responses to African Swine Fever Virus: Implications for Vaccine Development. Veterinary microbiology 249:108832.

Xian, Y, and C Xiao $\ 2020 \otimes T$ The Structure of ASFV Advances the Fight against the Disease. Trends in biochemical sciences 45(4):276-278.

Yang Q, Li G, Zhu Y, Liu L, Chen E, Turnquist H, Zhang X, Finn OJ, Chen X, Lu B囚2011 『lL-33 synergizes with TCR and IL-12 signaling to promote the effector function of CD8+ T cells. European journal of immunology 41(11):3351-60.

Yang WT, Yang GL, Yang X, Shonyela SM, Zhao L, Jiang YL, Huang HB, Shi CW, Wang JZ, Wang $₫ 2017 \rrbracket$ Recombinant Lactobacillus plantarum expressing HA2 antigen elicits protective immunity against H9N2 avian influenza virus in chickens. Applied microbiology and biotechnology 101:8475-8484.

\section{Figures}




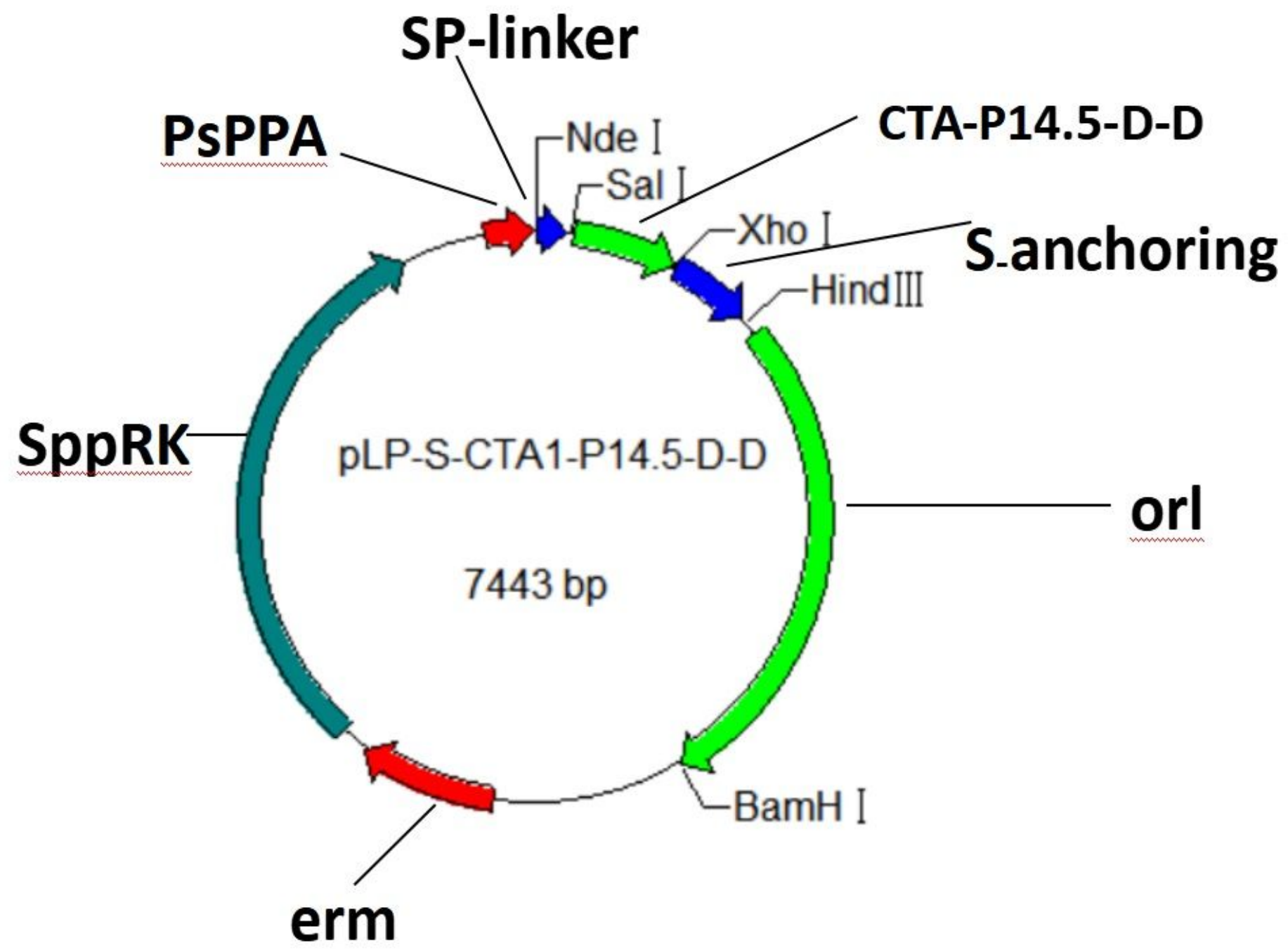

Figure 1

A囚a map of the plasmid NC8-pLP-S-p14.5;B囚a map of the plasmid NC8-pLP-S-p14.5-IL-33-Mus囚C:a map

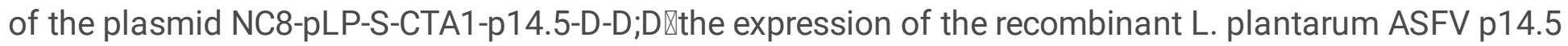
protein,p14.5-IL-33-Mus fusion protein and CTA1-p14.5-D-D fusion protein was verified by Western blot. 

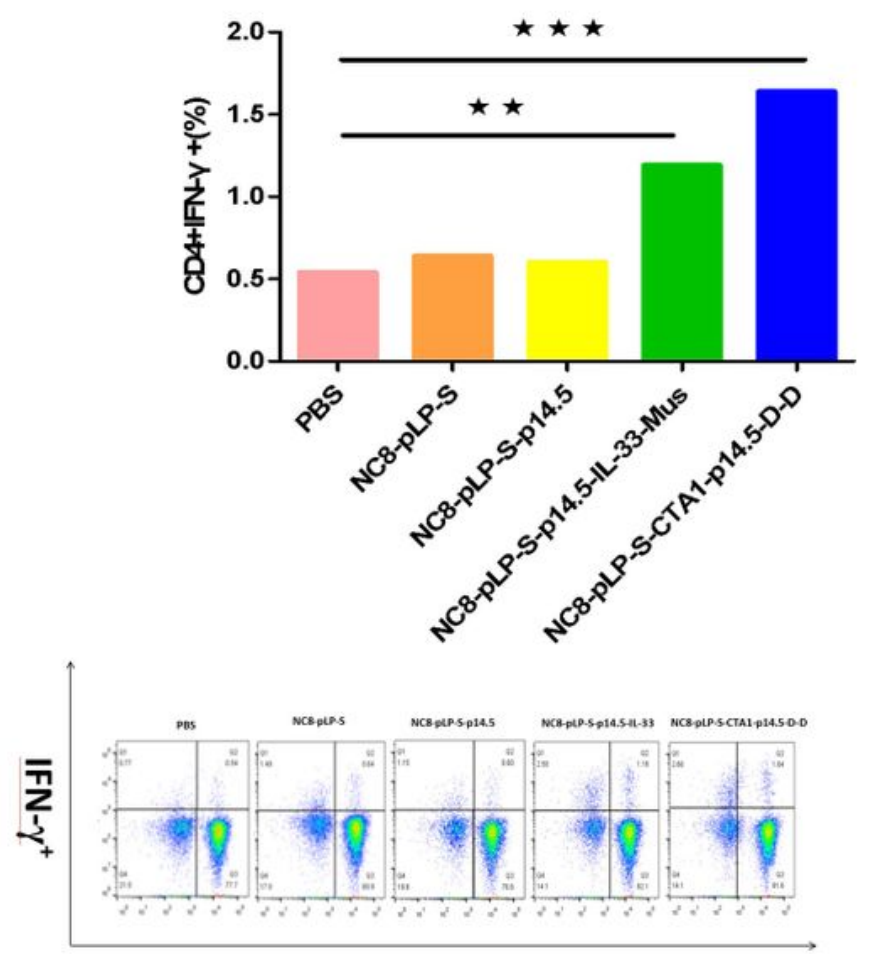

\section{$\mathrm{CD}^{+}$}
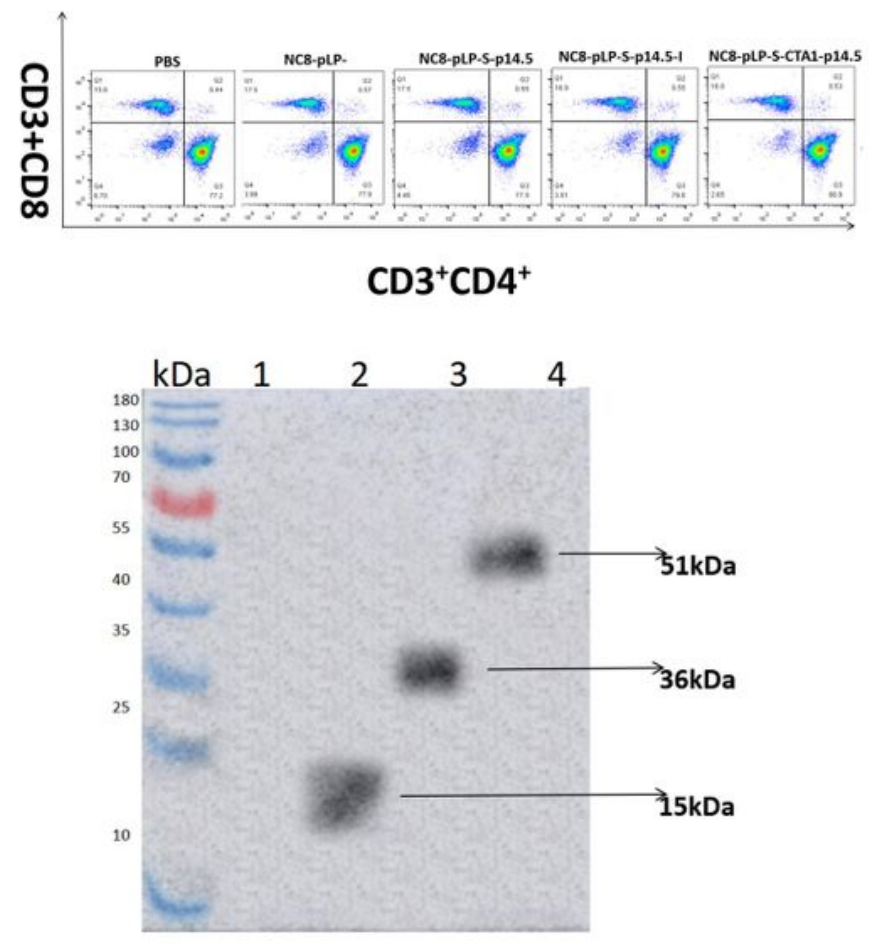

\section{Figure 2}

$A$ : Changes in the detection of $C D 3+C D 4+$ and $C D 3+C D 8+T$ cells in the spleen;B:Changes in the detection of CD3+ CD4+ and CD3+ CD8+ T cells in the mesenteric lymph nodes; $\mathrm{C}$ :changes in CD4+ IFN- $\gamma+$ in the spleen; $\mathrm{D} \llbracket$ changes in $\mathrm{CD} 8+\mathrm{IFN}-\gamma+$ in the spleen. $\mathrm{E} \llbracket$ Statistical analysis of lymphocyte expression of B220 and IgA in mouse PPs. 
Fecal SIgA

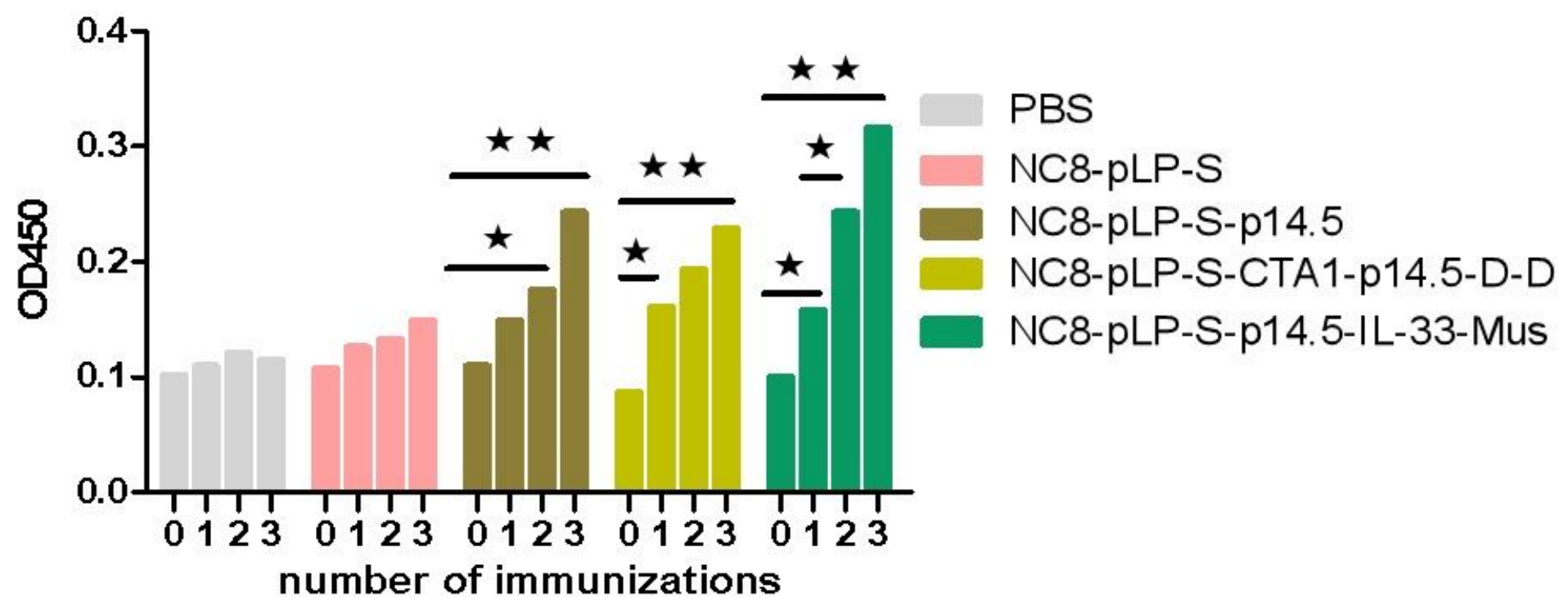

Figure 3

Comparison of the increase in fecal IgA content in the five groups of mice after three immunizations 